\title{
Polar molecules in frustrated triangular ladders
}

\author{
Tapan Mishra, Sebastian Greschner, and Luis Santos \\ Institut für Theoretische Physik, Leibniz Universität Hannover, 30167 Hannover, Germany
}

(Received 2 December 2014; published 13 April 2015)

\begin{abstract}
Polar molecules in geometrically frustrated lattices may result in a very rich landscape of quantum phases, due to the nontrivial interplay between frustration, and two- and possibly three-body intersite interactions. In this paper we illustrate this intriguing physics for the case of hard-core polar molecules in frustrated triangular ladders. Whereas commensurate lattice fillings result in gapped phases with bond order and/or density-wave order, at incommensurate fillings we find chiral, two-component, and pair superfluids. We show as well that, remarkably, polar molecules in frustrated lattices allow for the observation of bond-ordered supersolids.
\end{abstract}

DOI: 10.1103/PhysRevA.91.043614

PACS number(s): 67.85.-d, 75.40.Gb, 71.27.+a

\section{INTRODUCTION}

Ultracold quantum gases in optical lattices constitute an excellent scenario for the study of strongly correlated manybody systems [1], as highlighted by the superfluid (SF) to Mott-insulator transition of cold atoms in optical lattices [2] driven by the interplay between intersite hopping and twobody on-site interactions. Two-body intersite interactions are expected to play a crucial role in polar lattice gases, including magnetic atoms, polar molecules, and Rydberg atoms [3-5]. Intersite dipole-dipole interactions may result under proper conditions in crystalline and supersolid phases $[3,6]$.

In typical experiments up to now multibody interactions have played a negligible role compared to two-body ones. It has been recently proposed, however, that three-body nearest-neighbor interactions may be achieved under proper conditions in lattice gases of polar molecules [7] (for a recent proposal on how to induce three- and even four-body on-site interactions in alkali-metal lattice gases see Ref. [8]). The seminal proposal of Ref. [7] presented fundamental questions about the effect of intersite three-body interactions in lattice gases. In particular, recent works have shown that three-body interactions may result in supersolid and devil's staircase phases in two dimensions [9] and in a phase transition in one-dimensional (1D) systems from a SF to a gapped phase with simultaneous charge-density-wave (CDW) order and bond order (BO) [10].

Frustrated lattice systems constitute nowadays a very active research focus, due to recent experimental progress in the creation of synthetic gauge fields [11-13] and the possibility of realizing frustrated lattice geometries [14]. The rich physics resulting from the interplay between frustration and interactions has attracted growing theoretical attention [15-25]. Recently, experiments on dynamically frustrated optical lattices in the presence of a synthetic gauge field have investigated the onset of chirality by observing the Meissner to vortex-phase transition [26].

In this paper we are interested in the rich physics that results from the interplay between frustration and intersite interactions in frustrated polar lattice gases. We illustrate the physics that may result from that interplay by considering the particular case of hard-core polar molecules in frustrated triangular ladders. By means of extensive numerical calculations based on density-matrix renormalization-group techniques [27,28], we show that the frustrated polar system is characterized by the appearance of commensurate gapped phases with bond-order and/or density-wave order and by chiral, two-component, and pair superfluids at incommensurate filling. Moreover, we show that, remarkably, polar molecules in frustrated lattices may allow for the first realization of bond-ordered supersolids.

The structure of the paper is as follows. In Sec. II we introduce the lattice model we employ to illustrate the interplay of frustration and two- and three-body interactions in polar lattice gases. Sections III and IV discuss in detail the gapped and gapless phases, respectively, found in the ground-state phase diagram. Section V discusses the dilute limit, whereas Sec. VI discusses an effective quasiparticle model in the strongly interacting regime. Finally, in Sec. VII we briefly summarize our conclusions.

\section{MODEL}

We consider in the following a system of polar molecules in a frustrated triangular ladder as depicted in Fig. 1, characterized by hopping $t>0$ and $t^{\prime}<0$ along the rungs and the legs, respectively. The change in sign of $t^{\prime}$ may be achieved, e.g., by lattice shaking as recently realized experimentally [14]. Such a ladder is actually equivalent to a 1D lattice with nearestneighbor (NN) hopping $t$ and next-nearest-neighbor (NNN) tunneling $t^{\prime}$. As previously discussed, under proper conditions, polar molecules may present in addition to two-body intersite interactions, which we restrict in the following to the NN interaction $V$, significant three-body $\mathrm{NN}$ interactions $W$ of molecules belonging to the same triangular plaquette (see Fig. 1). In the following we explore in particular a simplified hard-core scenario, in which maximally one molecule may occupy each site. The latter condition assume large-enough on-site interactions and a lattice filling smaller than one. The Hamiltonian describing the system is of the form

$$
\begin{aligned}
H= & -t \sum_{i}\left(a_{i}^{\dagger} a_{i+1}^{\dagger}+\text { H.c. }\right)-t^{\prime} \sum_{i}\left(a_{i}^{\dagger} a_{i+2}+\text { H.c. }\right) \\
& +V \sum_{i} n_{i} n_{i+1}+W \sum_{i} n_{i} n_{i+1} n_{i+2}
\end{aligned}
$$

where $a_{i}^{\dagger}$ and $a_{i}$ are creation and annihilation operators for bosons at site $i$ and $n_{i}=a_{i}^{\dagger} a_{i}$ is the boson number operator at site $i$. We set below $t=1$ as the energy scale.

Spin systems with two-body interactions in such ladders have been studied extensively both numerically and 


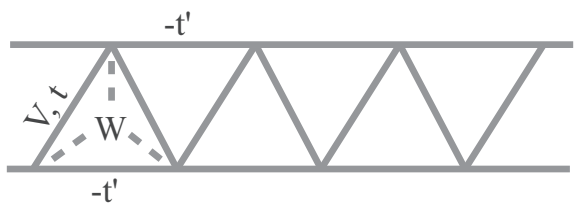

FIG. 1. Triangular ladder lattice with two- and three-body interactions $V$ and $W$, respectively. The hopping along the rungs and the legs are $t>0$ and $t^{\prime}<0$, respectively.

analytically [29-32], at half filling [33,34], and with magnetic fields in the ferromagnetic [35] and antiferromagnetic regimes [36,37]. Model (1) presents no exact solution except for $W=t^{\prime}=0$ for which there exists a SF to CDW phase transition at $V=2 t$ [38] and for $W=V=0$ for which the ground state can be obtained exactly at $t^{\prime}=-t / 2$ [24].

In this paper we perform an extensive numerical study of model (1) and in particular of the interplay between frustration and two- and three-body interactions as a function of the lattice filling $0<\rho<1$. We find a very rich landscape of ground-state phases, as illustrated in Fig. 2 for $t^{\prime}=$ -1 and $V=W$. For half filling $\rho=1 / 2$, the competition between frustration and two-body interactions results in a phase transition between a BO phase and a CDW phase, which is of first-order nature, as predicted in Ref. [24]. In contrast, for $\rho=2 / 3$ the three-body interactions result in a Berezinskii-Kosterlitz-Thouless (BKT) transition between a $\mathrm{SF}$ and a phase with both $\mathrm{BO}$ and CDW orders (the BO plus CDW phase) [10]. In addition to these phases, we find a wealth of gapless phases at incommensurate fillings. For

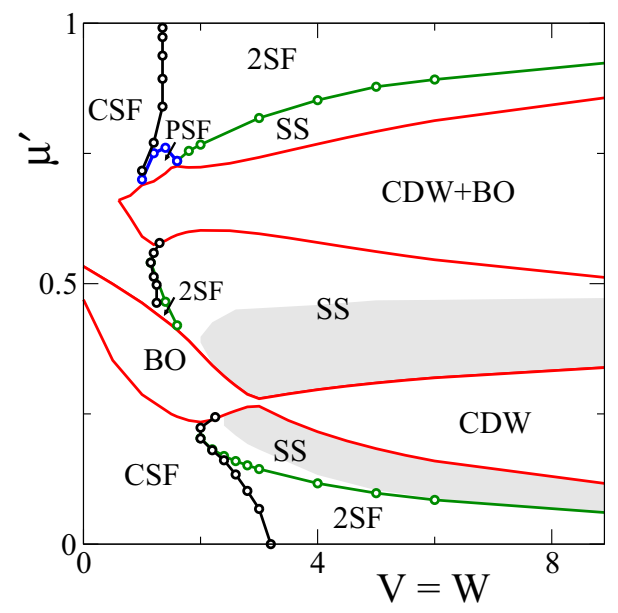

FIG. 2. (Color online) Phase diagram of model (1) as a function of $\mu^{\prime}$ and $V=W$ for $t^{\prime}=-t=-1$. Here $\mu^{\prime}=\frac{9+4 \mu}{20 W+18}$ is the scaled chemical potential such that $\mu^{\prime}=0$ (1) corresponds to the vacuum (full) state. Solid (red) curves mark the boundaries of gapped phases, such as BO and CDW phases at $\rho=1 / 2$ and BO plus CDW phases at $\rho=2 / 3$. The CSF phase is present for all $\mu^{\prime}$ (except for half filling) and weak interactions. The black circles separate the CSF phase from other superfluid phases. The 2SF-SS transition is marked by green circles. The shaded region corresponds to the CSS phase. The PSF phase is a small region appearing in the upper part of the BO plus CDW phase. small two- and three-body interactions and all fillings (except for half filling), the systems becomes a chiral superfluid (CSF) that carries a finite long-range current order [37]. This current order vanishes with growing interactions and the system enters into nonchiral SF phases, which include supersolid (SS), two-component superfluid (2SF), and pair-superfluid (PSF) phases. Interestingly, these three phases exhibit a coexisting nonvanishing peak in both $\mathrm{BO}$ and $\mathrm{CDW}$ structure factors. In the following section we discuss these bond-ordered supersolid phases in more detail.

\section{GAPPED PHASES}

We discuss in this and the next section in detail the phases depicted in Fig. 2 corresponding to the case $t^{\prime}=-1$ and $V=$ $W$. At half filling $\rho=1 / 2$, the system is characterized by the appearance of gapped incompressible BO and CDW phases. As shown in Fig. 2, these phases are separated by a first-order phase transition at $V=W \simeq 3.0$. The $\mathrm{BO}$ phase results from frustration that favors dimerization in the small- $V$ regime, whereas large $V$ favors the CDW phase [24]. Three-body interactions do not play any significant role in these phases since in them three molecules never coexist simultaneously at the same triangular plaquette. The BO phase is characterized by a finite bond-order structure factor

$$
S_{\mathrm{BO}}(k)=\frac{1}{L^{2}} \sum_{i, j} e^{i k(i-j)}\left\langle B_{i} B_{j}\right\rangle
$$

where $B_{i}=a_{i}^{\dagger} a_{i+1}+a_{i+1}^{\dagger} a_{i}$, whereas the CDW presents a finite value of the density-density structure factor

$$
S_{\mathrm{CDW}}(k)=\frac{1}{L^{2}} \sum_{i, j} e^{i k(i-j)}\left(\left\langle n_{i} n_{j}\right\rangle-\left\langle n_{i}\right\rangle\left\langle n_{j}\right\rangle\right)
$$

at the wave vector $k=\pi$. At the transition point the excitation gap diminishes, but never vanishes, showing the first-order nature of the phase transition.

For $\rho=2 / 3$ the system presents another gapped incompressible phase, $\mathrm{BO}$ plus $\mathrm{CDW}$, that possesses both $\mathrm{BO}$ and CDW orders simultaneously. In the absence of $t^{\prime}$ and $V$ the system undergoes a SF to BO plus CDW BKT transition at $W \sim 3.0$ [10] where the Luttinger liquid parameter $K=2 / 9$ and $S_{\mathrm{CDW}}(k)$ has a peak at $k=2 \pi / 3$. We observe that, in the presence of both frustration and intersite two-body interactions the $\mathrm{BO}$ plus $\mathrm{CDW}$ phase is enhanced and hence the SF to BO plus CDW transition occurs at a much smaller value $V=W \simeq 1.0$ (see Fig. 2). This happens because lattice frustration favors $\mathrm{BO}$ whereas two-body intersite interactions favor CDW order.

In order to distinguish between gapped and gapless phases we evaluate the chemical potential $\mu$ obtained from the minimization of $E(L, N)-\mu N$, where $E(L, N)$ is the groundstate energy of the system with $L$ sites and $N$ bosons [35]. In Fig. 3(a) we plot $\mu$ as a function of $\rho$ for $L=120$ and $V=W=2.6$. It can be seen that $\mu$ jumps at $\rho=1 / 2$ and $2 / 3$, indicating the gapped phases. 


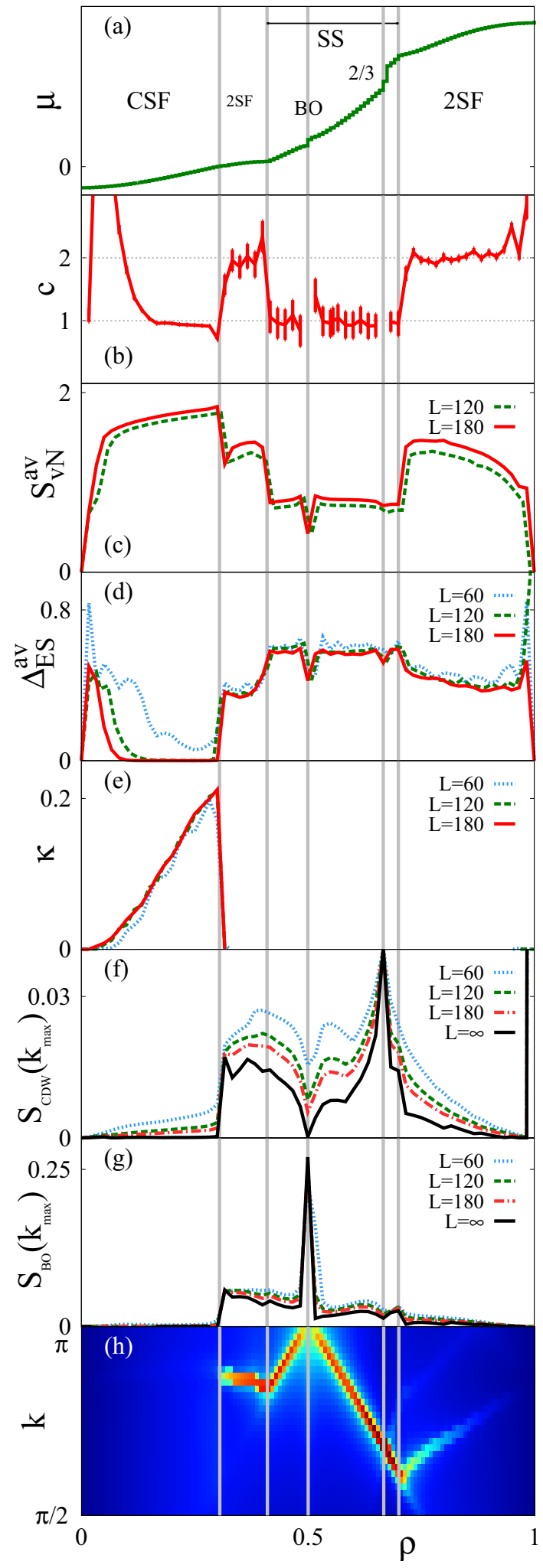

FIG. 3. (Color online) Cut through the phase diagram of Fig. 2 for $V=W=2.6$. We depict as a function of $\rho$ the (a) chemical potential $\mu(L=120)$, (b) central charge $c(L=180)$, (c) entanglement entropy, (d) entanglement gap, (e) chirality $\kappa$, (f) maximum of $S_{\mathrm{CDW}}(k)$, and $(\mathrm{g})$ maximum of $S_{\mathrm{BO}}(k)$ [both (f) and $(\mathrm{g})$ are obtained by means of a linear extrapolation to $L \rightarrow \infty$ using the two largest system sizes $L=120$ and 180]. (h) $S_{\mathrm{CDW}}(k)$ as a function of (quasi)momentum $k$ and filling $\rho(L=120)$.

\section{GAPLESS PHASES}

\section{A. 2SF phases}

For $\rho \neq 1 / 2$ or $2 / 3$, the system is characterized by different types of SF phases. In order to understand in more detail the nature of these phases, we consider a fixed $V=W=$ 2.6 and plot various relevant physical quantities as a function of $\rho$ in Fig. 3. As discussed above, $\mu(\rho)$ [Fig. 3(a)] shows the appearance of incompressible (gapped) and compressible (gapless) phases. A first differentiation between gapless phases is provided by the study of the von Neumann entropy

$$
S_{v N, L}(l)=-\operatorname{tr}\left(\rho_{l} \ln \rho_{l}\right)=\frac{c}{6} \ln \left[\frac{L}{\pi} \sin \left(\frac{\pi}{L} l\right)\right]+g,
$$

where $\rho_{l}$ is the reduced density matrix for a subsystem of length $l$ embedded in a chain of a finite length $L$ and the last equation is valid for conformally invariant gapless states $[39,40]$, with $c$ the central charge and $g$ a constant. Figure 3(b) shows the value of $c$ extracted by the finite-size scaling of $S_{v N, L}(l)$. We observed the appearance of regions with $c=1$ (SF phases with a single gapless mode) and regions with $c=2$ (and hence two gapless modes). We refer to the latter as $2 \mathrm{SF}$ phases [41].

Apart from estimating $c$, both entanglement entropy $S_{v N, l}$ and the spectrum of eigenvalues $s_{i}^{2}(l)$ of the reduced density matrix $\rho_{l}$ of the subsystem itself, the so-called entanglement spectrum, have been shown to offer a sensitive probe for quantum phase transitions [40,42-46]. We have computed in particular the gap in the entanglement spectrum $\Delta_{\mathrm{ES}}(l)=$ $\sum_{i}(-)^{i} s_{i}(l)$. In order to minimize boundary effects, we have averaged $S_{v N, L}(l)$ and $\Delta_{\mathrm{ES}}(l)$ over all bipartitions in the range $[L / 4,3 L / 4]$. We denote these averages by $S_{v N}^{\mathrm{av}}$ and $\Delta_{\mathrm{ES}}^{\mathrm{av}}$. The 2SF-SF transitions are marked by sharp jumps in $S_{v N}^{\mathrm{av}}$ and $\Delta_{\mathrm{ES}}^{\mathrm{av}}$ [see Figs. 3(c) and 3(d)]. Interestingly, these transitions are also characterized by a kink in the $\mu(\rho)$ plot of Fig. 3(a).

\section{B. Chiral superfluid phases}

Geometric frustration dominates the system for weak interactions $V=W$. In this regime, the frustration leads to a CSF phase for all values of $\rho$ (except $\rho=1 / 2$ ). The CSF phase eventually vanishes for sufficiently large interactions (Fig. 2). Chiral superfluid phases are characterized by a nonzero chiral order parameter

$$
\kappa=\lim _{|i-j| \gg 1}\left\langle\kappa_{i} \kappa_{j}\right\rangle,
$$

where $\kappa_{i}=i\left(a_{i}^{\dagger} a_{i+1}-a_{i+1}^{\dagger} a_{i}\right)$. As shown in Fig. 3(e), $\kappa>0$ for low fillings (the CSF phase) vanishes sharply at the onset of a $2 \mathrm{SF}$ phase and remains zero for all larger- $\rho$ values. The CSF phase is characterized by the spontaneous symmetry breaking of the $Z_{2}$ symmetry associated with the two dispersion minima induced by the lattice frustration [18]. This spontaneoussymmetry-breaking nature of the CSF phase becomes apparent from the vanishing $\Delta_{\mathrm{ES}}^{\mathrm{av}}$ [Fig. 3(d)] (note, however, that $\Delta_{\mathrm{ES}}^{\mathrm{av}}$ shows strong finite-size corrections especially at low $\rho$ ).

\section{Supersolid phases and bond-ordered supersolids}

Supersolids are characterised by the coexistence of CDW order and superfluidity. Supersolids can be achieved in bosonic lattice systems with nearest-neighbor interactions $[3,6]$. 
Whereas at commensurate densities the system undergoes for large-enough interactions a SF-CDW transition, a SS may be obtained by doping the system away from the crystalline phases. In one dimension, hard-core bosons do not exhibit supersolidity due to large fluctuations and the hard-core repulsion. However, kinetic frustration can contribute significantly to stabilize SS phases [23].

In contrast to the case of soft-core bosons, the SS phases in the hard-core case are characterized by a peak in $S_{\mathrm{CDW}}$ at a value $k_{\max }$ incommensurate with the lattice and dependent on the filling $\rho[24,47]$. As mentioned above, in the present case, there exist two CDW phases with $S_{\mathrm{CDW}}$ peaked at $k_{\max }=\pi$ $(\rho=1 / 2)$ and $2 \pi / 3(\rho=2 / 3)$. By doping the system away from these CDW phases, we retain a finite CDW order in the adjacent $S F$ region that interpolates between the $k_{\max }$ values in the CDW regions. Figure 3(h) shows the linear $\rho$ dependence of $k_{\max }$ in the superfluid regions adjacent to the CDW phases. The presence of crystalline order is confirmed by a finite value of $S_{\mathrm{CDW}}(k)$ in the thermodynamic limit [see Fig. 3(f)]. It can be seen that $S_{\mathrm{CDW}}(k)=0$ in both the CSF phase and the BO phase at $\rho=1 / 2$. The presence of CDW order in the superfluid phases confirms the existence of supersolidity (note that the 2SF has a finite CDW order and hence it is also a supersolid phase).

Remarkably, in addition to a finite CDW order we observe a finite coexisting bond order. Figure 3(g) depicts the maximum of $S_{\mathrm{BO}}(k)$ as a function of $\rho$. Clearly the system exhibits simultaneous CDW and BO orders not only at the gapped BO plus CDW order at $\rho=2 / 3$, but for incommensurate fillings within the superfluid region as well (except in the $\mathrm{CSF}$ region where both $\mathrm{BO}$ and $\mathrm{CDW}$ order vanish). We would like to mention that the bond ordering in the system of hard-core bosons arises due to the effect of frustrated nextnearest-neighbor hopping $t^{\prime}$ at low filling around $\rho=1 / 2$. However, in the high-filling regime, the bond ordering occurs due to the effect of the three-body interaction $W$. It can be recalled that in the absence of $t^{\prime}$ and $V$, the system undergoes a SF to BO plus CDW phase transition at $\rho=2 / 3$ filling [10]. The presence of additional $V$ only renormalizes the critical point of this transition. As we depart from these commensurate densities, the system exhibits bond ordering at large frustration and interaction. The BO plus SS phase is the result of both frustration and interaction in the model considered here.

\section{Commensurate and incommensurate supersolids}

Another interesting feature of the supersolid phases is revealed by the momentum distribution

$$
n(k)=\frac{1}{L} \sum_{i, j} e^{i k(i-j)}\left\langle a_{i}^{\dagger} a_{j}\right\rangle .
$$

Figure 4 depicts $n(k)$ for different $\rho$ for the same case of Fig. 3. While in the 2SF and CSF region we observe two peaks at incommensurate momenta $\pm k$ [18], in the SS region $n(k)$ significantly broadens. Interestingly, we find a wide SS region that still exhibits two maxima in $n(k)$, while in other regions of the SS phase $n(k)$ presents a single maximum at $k=0$. We call the former an incommensurate supersolid (ICSS) and the latter a commensurate supersolid (CSS). The CSS region (shaded area in Fig. 2) grows slowly with increasing $V=W$

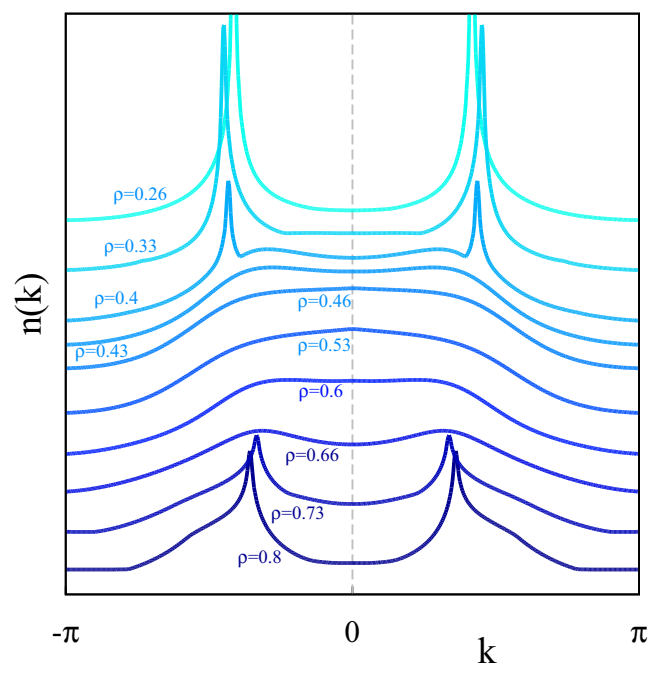

FIG. 4. (Color online) Momentum distribution $n(k)$ for different densities $(V=W=2.6, L=180)$. The single curves have been shifted for clarity. In the $2 \mathrm{SF}(\rho=0.33,0.4,0.73$, and 0.8$)$ and the CSF ( $\rho=0.26)$ phases, $n(k)$ exhibits two peaks at incommensurate momenta $\pm k$. When approaching the SS phase the momentum distribution broadens but still shows two maxima (for $\rho=0.4$, $0.43,0.6$, and 0.66 ), characterizing the ICSS region. However, at intermediate fillings ( $\rho=0.53$ ) only one maximum is observed (CSS region).

(e.g., we do not observe a single maximum for $\rho>2 / 3$ for $W=V \lesssim 20$ ). However, as we show below, in the limit of strong interactions only the CSS phase survives.

\section{E. Pair-superfluid phase}

Apart from the CSF and SS phases we observe a small pair-superfluid (PSF) region in the phase diagram of Fig. 2. Pair-superfluid phases are characterized by algebraically decreasing pair-pair correlations that coexists with exponentially decreasing single-particle correlations. We would like to mention that the PSF region gets enhanced as $\left|t^{\prime}\right|$ increases. As shown below, this may be also well understood in the limit of strong interactions.

\section{DILUTE LIMIT}

The CSF to 2SF transition is best understood in the dilute limit $\rho \rightarrow 0$ [48]. In this limit the single-particle dispersion in the triangular ladder develops two degenerate minima for $\left|t^{\prime} / t\right|>1 / 4$. While for a single-minimum dispersion the system is a one-component $\mathrm{SF}$, in the presence of two minima two different types of SFs may occur: Either the bosons equally occupy both minima, which corresponds to a $2 \mathrm{SF}$, or one of them is spontaneously selected. The latter is the case of the CSF, which hence exhibits a nonzero average momentum, a nonvanishing chiral current, and a sharp single peak in $n(k)$ away from $k=0$ in the thermodynamic limit [18].

As shown in [48], one may obtain quantitative insight in the competition between CSF and 2SF phases in the dilute limit from solving the low-energy scattering problem of two bosons on the triangular ladder. From the two-particle problem one may obtain different types of scattering solutions 


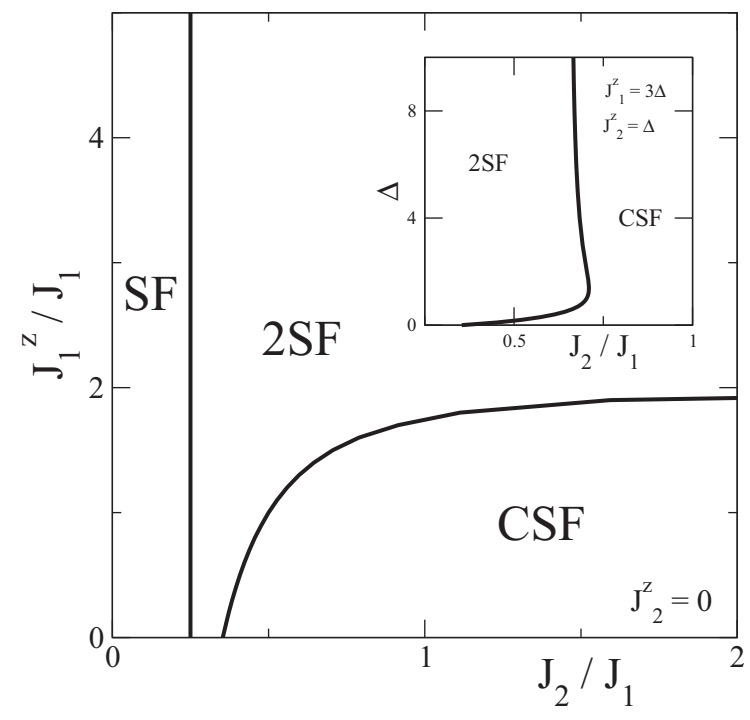

FIG. 5. Analytical prediction of the phase diagram for filling $\rho \rightarrow$ 0 as a function of the frustration $J_{2} / J_{1}$ and the interaction $\Delta=V=$ $W$. The inset shows the phase boundaries for $J_{1}^{z}=3 \Delta$ and $J_{2}^{z}=\Delta$, which correspond to the particular case of $\rho \rightarrow 1$ of model (1).

and extract two relevant scattering lengths: one for bosons belonging to the same single-particle minimum $a_{1,1}=a_{2,2}$ and other one for bosons belonging to different minima $a_{1,2}$. One can relate the 1D scattering length to the amplitude of the contact interaction potential of the two-component Bose gas of mass $m$ as $g_{i, j}=-2 / a_{i, j} m$. As the intracomponent interaction is stronger than the intercomponent interaction $g_{1,1}=g_{2,2}>g_{1,2}$ in the limit of vanishing density $\rho \rightarrow 0$, the $2 \mathrm{SF}$ phase is favored. For a dominant intercomponent interaction the particles would preferably occupy only one of the two minima that is spontaneously chosen and hence the system would be in the CSF phase.

The quantitative analysis may be performed closely along the lines of Ref. [48]. We may map the dilute bosonic model into a spin-1/2 model $(0,1 \rightarrow \uparrow, \downarrow)$. The Hamiltonian then becomes a $J_{1}-J_{2}$ model with NN and NNN $S^{z} S^{z}$ interactions

$$
\begin{aligned}
H_{1 / 2}^{\text {dilute }}= & J_{1} / 2 \sum_{i} S_{i}^{+} S_{i+1}^{-}+J_{2} / 2 \sum_{i} S_{i}^{+} S_{i+2}^{-}+\text {H.c. } \\
& +J_{1}^{z} \sum_{i} S_{i}^{z} S_{i+1}^{z}+J_{2}^{z} \sum_{i} S_{i}^{z} S_{i+2}^{z},
\end{aligned}
$$

where $S_{i}^{x, y, z}$ denote the spin operators associated with the site $i$. For the low-filling limit, the spin couplings are given by $J_{1}^{z}=V=W$ and $J_{2}^{z}=0$, whereas $J_{1} / 2=t$ and $J_{2} / 2=t^{\prime}$. The resulting phase diagram is shown in the main panel of Fig. 5. By increasing $J_{1}^{z}$ one observes the transition between the CSF and the 2SF phase for $J_{2} / J_{1}>1 / \sqrt{8}$. The estimated value $J_{1, c}^{z} / J_{1} \approx 1.8$, i.e., $V / t \approx 3.6$, for $J_{2}=J_{1}$ is consistent with our numerical estimates of the transition taken at finite density.

Note that at high fillings just below saturation $\rho \rightarrow 1$, we may again describe the system with the model (7). However, due to the broken particle-hole symmetry of model (1), the effective spin model presents different spin couplings $J_{1}^{z}=3 \Delta$ and $J_{2}^{z}=\Delta$ with $\Delta=V=W$. As shown in the inset of Fig. 5 , the CSF to 2SF transition at large fillings would be predicted for much lower values of $J_{2} / J_{1}$. However, in our numerical calculation we still find such a transition at $t=t^{\prime}$ for high filling, which corresponds to the discrepancy already reported in Ref. [48] for spin- $\frac{1}{2}$ systems with large values of $J_{1}^{z}$ and $J_{2}^{z}$.

\section{LIMIT OF STRONG INTERACTIONS}

An interesting insight into the properties of the system is provided by the analysis of the strongly interacting regime $V=W \gg t, t^{\prime}$ (still assuming hard-core bosons). Let us first consider the case of low filling $\rho<1 / 2$, where three-body interactions play a negligible role. In that regime we may identify pairs of subsequent $|01\rangle$ particles as $|1\rangle_{\text {eff }}$ and the

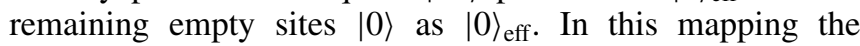
number of sites of the effective hard-core bosons is reduced to $L^{\prime}=L-N$ and the total density of the effective model $\rho^{\prime}$ fulfills $\rho=\frac{1}{1 / \rho^{\prime}+1}$. To first order in $\left(t, t^{\prime}\right) / V$, the effective quasiparticle model is given by an interaction-free $J_{1}-J_{2}$ Hamiltonian (similar models have been studied on square lattices in Refs. [49,50])

$$
H_{\mathrm{eff}}=J_{1} \sum_{i} c_{i}^{\dagger} c_{i+1}+J_{2} \sum_{i} c_{i}^{\dagger}\left(1-c_{i+1}^{\dagger} c_{i+1}\right) c_{i+2}+\text { H.c. }
$$

where $c^{\dagger}(c)$ are creation (annihilation) operators for the effective quasiparticles. The correlated NNN tunneling in Eq. (8) stems from the fact that a hopping to site $i+2$ is only allowed if there is no neighboring quasiparticle $|1\rangle_{\text {eff }}$ on site $i+1$. Model (8) also applies in the high-filling regime $\rho>2 / 3$. In that case, we may identify tuples of three sites $|011\rangle \rightarrow|1\rangle_{\text {eff }}$ and the remaining occupied sites $|1\rangle$ as $|0\rangle_{\text {eff }}$. The effective length reduces to $L^{\prime}=L-2 N$ and the density is mapped as $\rho=1-\frac{1}{1 / \rho^{\prime}+2}$. The phase diagram of model (8), depicted in Fig. 6, shows a SF-2SF transition for a critical $J_{2} / J_{1}<2$. We do not find any region with finite chirality. The SF phase of model (8) may be identified with the SS

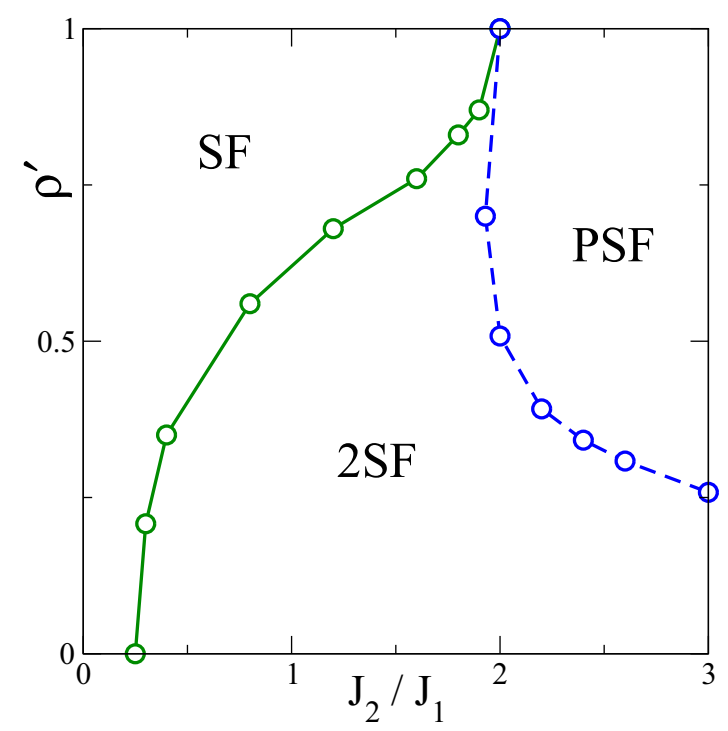

FIG. 6. (Color online) Phase diagram of model (8) as a function of $J_{2} / J_{1}$ and the effective density $\rho^{\prime}$. 


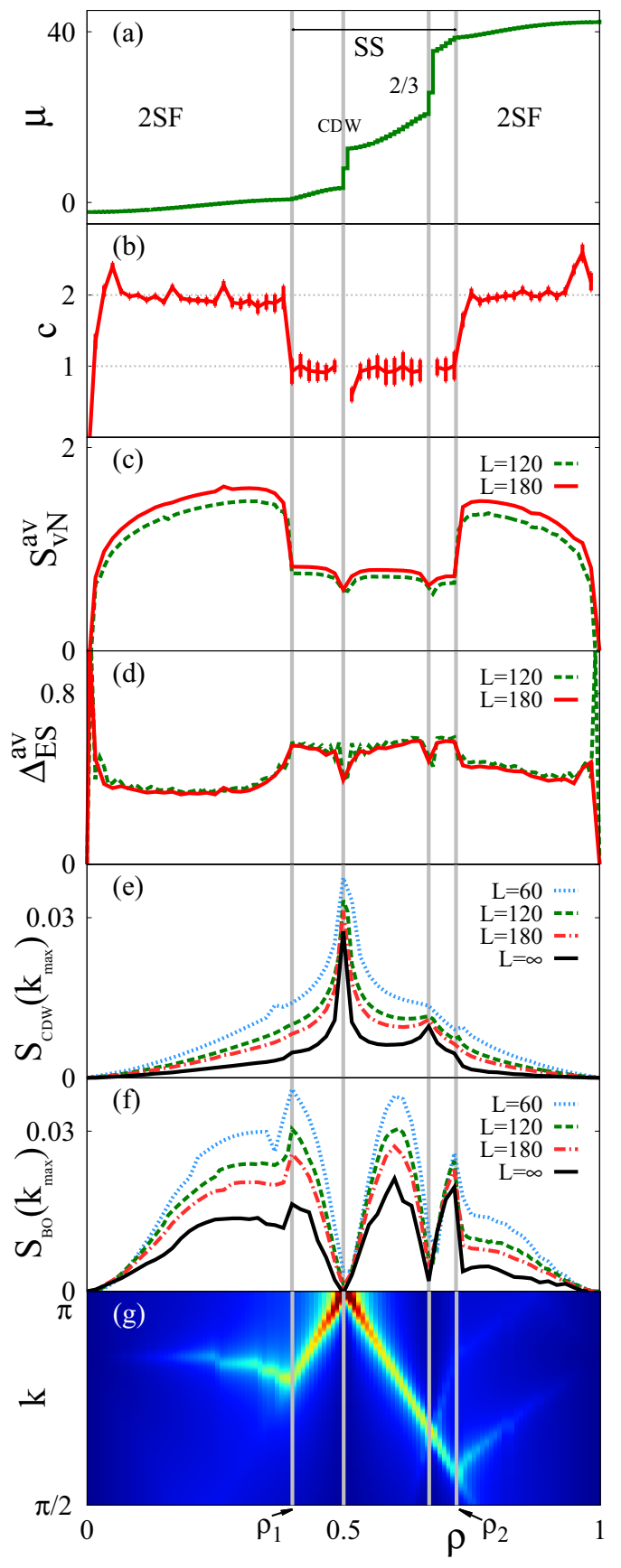

FIG. 7. (Color online) Cut through the phase diagram of Fig. 2 at large interactions, $V=W=8.0$, and $\left|t^{\prime}\right|=t=1$. We depict as a function of $\rho$ the (a) chemical potential $\mu(L=120)$, (b) central charge $c(L=180)$, (c) entanglement entropy, (d) entanglement gap, (e) maximum of $S_{\mathrm{CDW}}(k)$, and (f) maximum of $S_{\mathrm{BO}}(k)$. (g) Structure factor $S_{\mathrm{CDW}}(k)$ as a function of (quasi)momentum $k$ and $\rho(L=120)$.

phase of the original model due to the structure of the effective quasiparticles.

In Fig. 7 we depict our numerical results obtained for the original model (1) in the regime of large interactions $(V=$ $W=8$ and $\left.\left|t^{\prime}\right|=t=1\right)$. These numerical results agree well with those obtained from the strongly interacting quasiparticle model (8). For low and large fillings $(\rho<1 / 2$ and $\rho>3 / 2)$ we observe a $2 \mathrm{SF}$ to SF transition, clearly revealed by the central charge $c$ and the entanglement properties $\left(S_{V n}^{\mathrm{av}}\right.$ and $\left.\Delta_{\mathrm{ES}}^{\mathrm{av}}\right)$. The critical density for the SF-2SF transition obtained from the effective model matches well with the numerical estimates resulting from model (1). Figures 7 show SF-2SF transitions at $\rho_{1} \approx 0.4$ and $\rho_{2} \approx 0.72$, which agree well with $\rho_{c}^{\prime} \approx 0.64$. For all incommensurate densities $S_{\mathrm{CDW}}(k)$ and $S_{\mathrm{BO}}(k)$ exhibit a maximum at $k \neq 0$ that extrapolates to finite values in the thermodynamic limit (the extrapolation has been performed with polynomials of first and second order in $1 / L$ ).

As shown in Fig. 6, for large values of the NNN hopping $J_{2}$ it is energetically favorable at high densities to create pairs of holes (the PSF phase), because the correlated NNN hopping of isolated quasiholes is suppressed at high densities. This PSF phase at large NNN hoppings is connected to the small PSF region shown in Fig. 2 and will occupy larger regions of the phase diagram with increasing $t^{\prime}$. A similar situation has been recently studied for the case of low fillings in strongly interacting dipolar lattice gases [51].

For the particular choice of interactions $V=W$ the region of intermediate fillings $1 / 2<\rho<2 / 3$ in the large interaction limit may be mapped to a simple model of noninteracting hard-core particles. By adding single particles on top of the perfect $\rho=1 / 2 \mathrm{CDW}$ phase one creates two domain-wall excitations that behave again as particle pairs $|11\rangle$ of two sites. However, this pair may only move by single sites with some amplitude $J$. It is precisely the large three-body interaction $W$ that creates an effective hard-core repulsion of these excitations. Analogously, one can start the description on the background of the perfect $2 / 3$ crystalline phase. Hence, the large interaction limit of the intermediate fillings is described by a simple noninteracting spinless fermion model on a chain, explaining why in the large interaction limit all SS regions map to a one-component standard SF phase. This phase exhibits a single maximum in the momentum distribution, broadened due to the size of the effective quasiparticles and thus at large interactions only the CSS phase is present.

\section{CONCLUSION}

Polar molecules in geometrically frustrated lattices present a rich physics stemming from the interplay between frustration and two-body and possibly three-body intersite interactions. We have illustrated this physics for the particular case of hard-core polar molecules in frustrated triangular ladders. In addition to gapped phases at $\rho=1 / 2$ and $2 / 3$ filling, we have revealed the appearance of a wealth of incommensurate superfluid phases, including chiral superfluids, two-component superfluids, pair superfluids, and commensurate and incommensurate supersolids. Moreover, we have shown that except for the chiral superfluid phase, all superfluid phases exhibit coexisting bond and density-wave orders.

Although we have just considered the specific case of model (1), our findings point to general features for models of polar molecules in the presence of geometric frustration, in particular the competition of CSF and 2SF phases, and the appearance of bond-ordered supersolids due to the simultaneous effect of frustration and intersite interactions. Note in particular that three-body interactions play a negligible role at low fillings 
$\rho<1 / 2$ such that the lower part of the phase diagram of Fig. 2 resembles that of the $t-t^{\prime}-V$ model [23,24].

Finally, note that the predicted phases may be detected with state-of-the-art techniques in optical lattice experiments. The chiral and nonchiral phases may be distinguished by vanishing and appearing peaks in time-of-flight images [14]. Kinks in the tiered structure (in the presence of an overall harmonic confinement) may be employed to monitor phase transitions into SS phases, which may be also revealed by a broadening of the time-of-flight peaks. Plateaus in the tiered profile would show the commensurate-incommensurate transitions between gapless and gapped phases.

\section{ACKNOWLEDGMENTS}

We acknowledge support from the Center for Quantum Engineering and Space Time Research and the Deutsche Forschungsgemeinschaft (Research Training Group 1729). Computer simulations were carried out on the cluster system of the Leibniz Universität Hannover.
[1] M. Lewenstein, A. Sanpera, and V. Ahufinger, Ultracold Atoms in Optical Lattices (Oxford University Press, Oxford, 2012).

[2] M. Greiner, O. Mandel, T. Esslinger, T. W. Hänsch, and I. Bloch, Nature (London) 415, 39 (2002).

[3] T. Lahaye, C. Menotti, L. Santos, M. Lewenstein, and T. Pfau, Rep. Prog. Phys. 72, 126401 (2009).

[4] K.-K. Ni, S. Ospelkaus, M. H. G. de Miranda, A. Pe'er, B. Neyenhuis, J. J. Zirbel, S. Kotochigova, P. S. Julienne, D. S. Jin, and J. Ye, Science 322, 231 (2008).

[5] T. F. Gallagher and P. Pillet, in Advances in Atomic, Molecular, and Optical Physics, edited by E. Arimondo, P. R. Berman, and C. C. Lin (Academic, London, 2008), Vol. 56, p. 161.

[6] M. A. Baranov, M. Dalmonte, G. Pupillo, and P. Zoller, Chem. Rev. 112, 5012 (2012).

[7] H. P. Büchler, A. Micheli, and P. Zoller, Nat. Phys. 3, 726 (2007).

[8] D. S. Petrov, Phys. Rev. A 90, 021601(R) (2014).

[9] K. P. Schmidt, J. Dorier, and A. M. Läuchli, Phys. Rev. Lett. 101, 150405 (2008).

[10] B. Capogrosso-Sansone, S. Wessel, H. P. Büchler, P. Zoller, and G. Pupillo, Phys. Rev. B 79, 020503(R) (2009).

[11] Y.-J. Lin, R. L. Compton, K. Jiménez-García, J. V. Porto, and I. B. Spielman, Nature (London) 462, 628 (2009).

[12] M. Aidelsburger, M. Atala, M. Lohse, J. T. Barreiro, B. Paredes, and I. Bloch, Phys. Rev. Lett. 111, 185301 (2013).

[13] H. Miyake, G. A. Siviloglou, C. J. Kennedy, W. C. Burton, and W. Ketterle, Phys. Rev. Lett. 111, 185302 (2013).

[14] J. Struck, C. Ölschlger, R. Le Targat, P. Soltan-Panahi, A. Eckardt, M. Lewenstein, P. Windpassinger, and K. Sengstock, Science 333, 996 (2011).

[15] E. Orignac and T. Giamarchi, Phys. Rev. B 64, 144515 (2001).

[16] A. Dhar, M. Maji, T. Mishra, R. V. Pai, S. Mukerjee, and A. Paramekanti, Phys. Rev. A 85, 041602(R) (2012).

[17] A. Dhar, T. Mishra, M. Maji, R. V. Pai, S. Mukerjee, and A. Paramekanti, Phys. Rev. B 87, 174501 (2013).

[18] S. Greschner, L. Santos, and T. Vekua, Phys. Rev. A 87, 033609 (2013).

[19] A. Petrescu and K. Le Hur, Phys. Rev. Lett. 111, 150601 (2013).

[20] M. P. Zaletel, S. A. Parameswaran, A. Rüegg, and E. Altman, Phys. Rev. B 89, 155142 (2014).

[21] W. S. Cole, S. Zhang, A. Paramekanti, and N. Trivedi, Phys. Rev. Lett. 109, 085302 (2012).

[22] A. Dhar, T. Mishra, R. V. Pai, S. Mukerjee, and B. P. Das, Phys. Rev. A 88, 053625 (2013).
[23] T. Mishra, R. V. Pai, and S. Mukerjee, Phys. Rev. A 89, 013615 (2014).

[24] T. Mishra, R. V. Pai, S. Mukerjee, and A. Paramekanti, Phys. Rev. B 87, 174504 (2013).

[25] M. Piraud, F. Heidrich-Meisner, I. P. McCulloch, S. Greschner, T. Vekua, and U. Schollwck, arXiv:1409.7016.

[26] M. Atala, M. Aidelsburger, M. Lohse, J. T. Barreiro, B. Paredes, and I. Bloch, Nat. Phys. 10, 588 (2014).

[27] S. R. White, Phys. Rev. Lett. 69, 2863 (1992).

[28] U. Schollwoeck, Rev. Mod. Phys. 77, 259 (2005).

[29] A. K. Kolezhuk, Prog. Theor. Phys. Suppl. 145, 29 (2002); Phys. Rev. B 62, R6057 (2000).

[30] T. Vekua, A. Honecker, H.-J. Mikeska, and F. Heidrich-Meisner, Phys. Rev. B 76, 174420 (2007).

[31] T. Hikihara. M. Kaburagi, H. Kawamura, and T. Tonegawa, J. Phys. Soc. Jpn. 69, 259 (2000).

[32] T. Hikihara, J. Phys. Soc. Jpn. 71, 319 (2002).

[33] T. Hikihara, M. Kaburagi, and H. Kawamura, Phys. Rev. B 63, 174430 (2001).

[34] S. Furukawa, M. Sato, and S. Onoda, Phys. Rev. Lett. 105, 257205 (2010).

[35] T. Hikihara, L. Kecke, T. Momoi, and A. Furusaki, Phys. Rev. B 78, 144404 (2008)

[36] T. Hikihara and A. Furusaki, Phys. Rev. B 69, 064427 (2004).

[37] T. Hikihara, T. Momoi, A. Furusaki, and H. Kawamura, Phys. Rev. B 81, 224433 (2010).

[38] M. A. Cazalilla, R. Citro, T. Giamarchi, E. Orignac, and M. Rigol, Rev. Mod. Phys. 83, 1405 (2011).

[39] G. Vidal, J. I. Latorre, E. Rico, and A. Kitaev, Phys. Rev. Lett. 90, 227902 (2003).

[40] P. Calabrese and J. Cardy, J. Stat. Mech.: Theory Exp. (2004) P06002.

[41] The fit of Eq. (4) to the block-entanglement data of finite systems with open boundary conditions is typically not very precise. However, we find that the fits to the numerical data for systems of length $L=180$ quite accurately yield the values of the central charge $c=1$ in the SF phase and $c=2$ in the $2 \mathrm{SF}$ phases, as shown in Fig. 3(b). For low fillings we observe strong finite-size corrections and the estimated values of $c$ apparently diverge.

[42] H. Li and F. D. M. Haldane, Phys. Rev. Lett. 101, 010504 (2008)

[43] F. Pollmann, A. M. Turner, E. Berg, and M. Oshikawa, Phys. Rev. B 81, 064439 (2010). 
[44] D. Poilblanc, Phys. Rev. Lett. 105, 077202 (2010).

[45] X. Deng and L. Santos, Phys. Rev. B 84, 085138 (2011).

[46] G. De Chiara, L. Lepori, M. Lewenstein, and A. Sanpera, Phys. Rev. Lett. 109, 237208 (2012).

[47] D. Rossini, V. Lante, A. Parola, and F. Becca, Phys. Rev. B 83, 155106 (2011).
[48] A. K. Kolezhuk, F. Heidrich-Meisner, S. Greschner, and T. Vekua, Phys. Rev. B 85, 064420 (2012).

[49] K. P. Schmidt, J. Dorier, A. M. Läuchli, and F. Mila, Phys. Rev. Lett. 100, 090401 (2008).

[50] F. Mila, J. Dorier, and K. P. Schmidt, Prog. Theor. Phys. Suppl. 176, 355 (2008).

[51] H. Weimer, New J. Phys. 16, 093040 (2014). 